\title{
Learner Modelling Through Analyzing Cognitive Skills and Learning Styles *
}

\author{
Sabine Graf and Kinshuk \\ ${ }^{1}$ Vienna University of Technology, Women's Postgraduate College for \\ Internet Technologies, Vienna, Austria, sabine.graf@ieee.org \\ ${ }^{2}$ Athabasca University, School of Computing and Information Systems, \\ Athabasca, Canada, kinshuk@ieee.org
}

\begin{abstract}
Providing adaptivity in web-based educational systems supports learners in their learning process and makes learning easier for them. However, providing adaptivity requires knowing the needs of the learners. Therefore learner modelling, the process of building and updating a learner model, is a crucial aspect of adaptive systems and necessary for providing course content that fits to the learners' needs. In this chapter, we discuss recent research focusing on cognitive traits and learning styles. We show approaches by which cognitive traits and learning styles can be identified. In order to get additional data for improving learner modelling, we discuss investigations about the relationship of cognitive traits and learning styles, and the aspects of adaptation resulting from this combination.
\end{abstract}

\subsection{Introduction}

Different learners have different needs. They differ, for example, in their learning goals, their knowledge about the domain, their cognitive traits, and their learning styles. These individual differences affect the learning process and are the reasons why some learners find it easy to learn in a particular course whereas others find the same course difficult (Jonassen and Grabowski 1993).

* This research has been partly funded by the Austrian Federal Ministry for Education, Science, and Culture, and the European Social Fund (ESF) under grant 31.963/46-VII/9/2002. 
Several studies have been conducted considering individual differences of learners and their impact on learning and on the achievement of learners (for an overview see Jonassen and Grabowski 1993). As Jonassen and Grabowski summarized (1993), prior knowledge is one of the strongest and consistent individual difference predictor of learners' achievement. Although prior knowledge seems to account for more variance in learning than other individual differences, more recently educational researchers have focused on aspects of personal characteristics and abilities such as cognitive traits and learning styles and are investigating their impact on learning. These investigations are motivated by educational and psychological theories. For example, the cognitive load theory (Paas et al. 2003; Sweller 1988) suggests that learning happens best under conditions that are aligned with human cognitive architecture. Therefore, it is beneficial to incorporate the differences in cognitive architecture in order to avoid cognitive overload and thus a negative effect on learning. Regarding learning styles, Felder, for example, pointed out that learners with a strong preference for a specific learning style might have difficulties in learning if their learning style is not supported by the teaching environment (Felder and Silverman 1988; Felder and Soloman 1997). On the other hand, incorporating learning styles makes learning easier and leads to better achievement.

Individual differences impact learning in general, and hence play also an important role for technology enhanced learning. Adaptive systems address exactly this issue and aim at incorporating the different needs of learners by providing adapted courses. Brusilovsky, for example, defined adaptive systems as "systems which reflect some features of the user in the user model and apply this model to adapt various visible aspects of the system to the user" (Brusilovsky 1996, p 88). Considering this definition from the viewpoint of an adaptive educational system, the adaptation process in such a system consists of two parts: first, a model of the learner has to be built (and updated) which includes all necessary information about the learner to provide adaptivity and second, this information has to be used in order to generate adapted courses.

The learner model plays a crucial role in adaptive systems. The information included in the learner model is based on the system's beliefs about the learner. The process of building and updating the learner model is called learner modelling. While Self (1994) provided a comprehensive description of learner modelling from a point of view of the formal techniques, Brusilovsky $(1994,1996)$ classified learner models and techniques for learner modelling based on existing systems.

Brusilovsky (1996) distinguished between two different ways of learner modelling: automatic and collaborative learner modelling. In the former, 
the process of building and updating the learner model is done automatically based on the action of the learner when he/she is using the system for learning. The main problem with this approach is to get enough reliable information to build a robust student model. According to Brusilovsky (1996), a solution to this problem might be the use of additional, more reliable sources such as the results of tests, in the learner modelling process. On the other hand, in collaborative learner modelling the learner provides explicit feedback which can be used to build or update the learner model. For instance, the learner can provide data for the learner modelling mechanism such as stating explicitly whether a page was relevant for his/her learning goal. Another option is to let the learner do the adaptation by himself/herself and therefore show directly what he/she expects from the system. For example, the order of links on a page can be changed by the learner, showing the preferred order to the system. Another possibility is that the learner is allowed to directly update the information of the learner model.

In a learner model, different kinds of information can be included. Brusilovsky (1994) distinguished two major groups, namely models of course knowledge and models of individual subject-independent characteristics. Both are different in terms of the form of representation of the model as well as the methods used in its construction and application. While first investigations about learner modelling were focused on models about the course knowledge, more and more research is now done on modelling individual characteristics of learners.

In the following sections, we focus on two individual characteristics, namely cognitive traits and learning styles, and describe recent research that deals with detecting such information in order to build a learner model. As discussed before, an important issue of learner modelling is to get enough reliable information about the learner which can be addressed by the use of additional information. Concerning this matter, we discuss the relationship between cognitive traits and learning styles and show how this relationship can be used to improve learner modelling. Since an aim of learner modelling is to use the gathered information to provide adaptivity, the chapter also describes how this information can be used and gives some examples on how learners can be supported by adaptive systems.

\subsection{Identifying Individual Differences}

Identifying the individual differences and using this information to build and update a learner model are requirements for providing suitable 
adaptivity regarding the respective individual differences. The more information is available the more reliable is the learner model and the better the adaptation suit. In the following two subsections, we introduce collaborative and automatic approaches to identify cognitive traits and learning styles. Subsequently, we discuss how both of these characteristics are related to each other and how information about this relationship can be used to improve learner modelling and subsequent adaptation.

\subsubsection{Identifying Cognitive Traits}

The Cognitive Trait Model (CTM) (Kinshuk and Lin 2004; Lin and Kinshuk 2005) is a learner model that profiles learners according to their cognitive traits. Four cognitive traits, working memory capacity, inductive reasoning ability, information processing speed, and associative learning skills are included in CTM so far. The CTM offers the role of 'learning companion', which can be consulted by and interacted with different learning environments about a particular learner. The CTM can still be valid after a long period of time due to the more or less persistent nature of cognitive traits of human beings (Deary 2004). When a student encounters a new learning environment, the learning environment can directly use the CTM of the particular student, and does not need to "re-learn the student".

\section{Collaborative Learner Modelling Approach}

With regards to the collaborative learner modelling approach, information about the learners' cognitive traits can be gathered by asking them to perform tests to measure their cognitive traits. The gathered information from the tests can then be used by the CTM in order to provide adaptivity. An example for such a test is the WebOSPAN task (Graf et al. 2006), which is an online task developed to identify working memory capacity.

A drawback of the collaborative learner modelling approach is that the learners have some additional effort. In the case of cognitive traits, these tests take quite long time and special conditions are required, for example, asking the learners that they have no interruption during a test.

\section{Automatic Learner Modelling Approach}

In contrast to the collaborative learner modelling approach, the automatic learner modelling approach collects data from the behaviour of the learner while he/she is using the educational system. Therefore, data about the cognitive traits of each learner can be collected without any additional effort of the learners. 
Lin and Kinshuk (2005) describe a typical structure of an automatic learner modelling approach for the CTM (figure 1). The learner interface provides a presentation of the learning environment to interact with the learner. The Interface Listener Component is a mechanism that can monitor events created by learner's interactions with a learning environment. Learner interactions are interpreted as a series of learner actions performed on knowledge objects. Actions are then passed on to the Action History Components and are stored in Action History.

The performance-based model typically exists independently in the adaptive educational system. It represents learner's domain competence and models the problem-solving process that the learner undertakes. Certain learner behaviours, called Manifestation of Traits (MOTs), can be used to infer about the cognitive capacity. Information of the performancebased model, such as passing or failing a unit, can be useful for detecting MOTs of some cognitive traits, and therefore data in the performancebased model is used as a source by the MOT Detector Component.

Various MOTs are defined for each cognitive trait. Each MOT is a piece of an interaction pattern that manifests a learner's characteristics (e.g. low inductive reasoning ability). The MOT Detector Component contains knowledge about a number of MOTs and detects those MOTs within a series of actions that are requested from the Action History Component.

The Individualised Temperament Network Component (for a detailed description, see Lin and Kinshuk 2004) is responsible for calculating the cognitive traits of the learners which are passed through the Trait Model Gateway and saved in the Cognitive Trait Model.

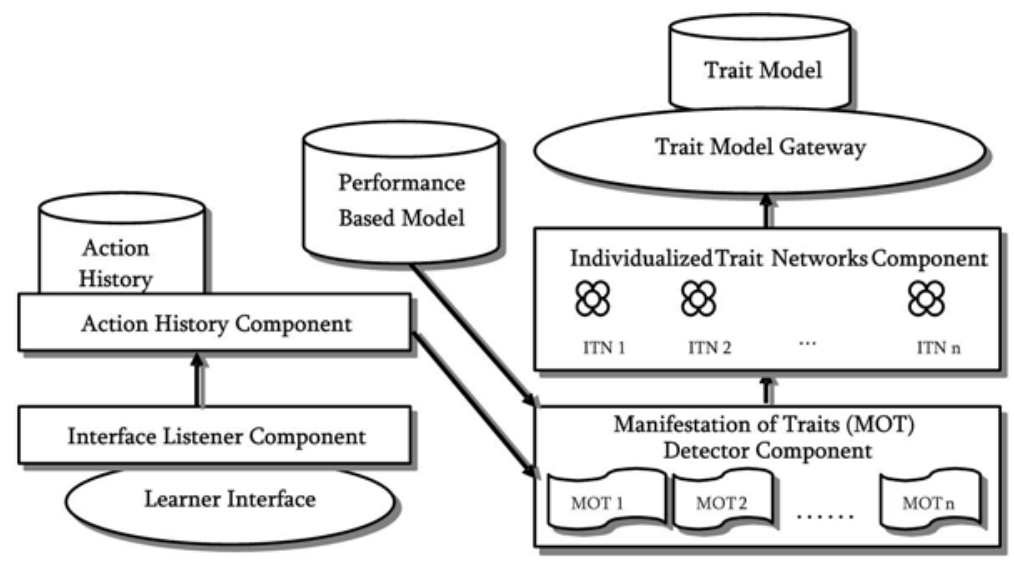

Fig. 1. Structural view of Cognitive Trait Model 


\subsubsection{Identifying Learning Styles}

Several different learning style models exist in the literature, each proposing different descriptions and classifications of learning types. These include the model by Kolb (1984), Honey and Mumford (1982), and Felder and Silverman (1988). Looking at adaptive educational systems incorporating learning styles, Felder-Silverman learning style model (FSLSM) is one of the most often used model and some researchers even argue that FSLSM is the most appropriate model for the use in adaptive web-based educational systems (e.g., Carver et al. 1999; Kuljis and Liu 2005). As a consequence, a lot of research work related to FSLSM deals with aspects on learner modelling and adaptivity. For this reason, the discussion about identifying learning styles is focused on FSLSM as well.

According to FSLSM, learners are characterized by their preferences in four bipolar dimensions: active learners learn by trying things out and working with others whereas reflective learners learn by thinking things through and prefer to work alone. Sensing learners like to learn concrete material and tend to be practical whereas intuitive learners prefer to learn abstract material such as theories and their meanings and tend to be more innovative than sensing learners. Visual learners remember best what they have seen whereas verbal learners get more out of words, regardless of whether they are spoken or written. Sequential learners learn in linear steps and prefer to follow linear stepwise paths whereas global learners learn in large leaps and are characterized as holistic. Each learner has a preference for each of these four dimensions.

\section{Collaborative Learning Modelling Approach}

For identifying learning styles, most existing adaptive systems have used a collaborative approach by asking learners to fill out a questionnaire. To detect learning styles according to FSLSM, the Index of Learning Styles (ILS) was developed (Felder and Soloman 1997). ILS consists of 44 questions and is available online. As a result, it provides four values between +11 and -11 , one for each dimension. Using the active/reflective dimension as an example, the value of " +11 " means that a learner has a strong preference for active learning, whereas the value of "-11" indicates that a learner has a strong preference for reflective learning.

The ILS is an often used instrument. Several kinds of investigations have been done on ILS data. Felder and Spurlin (2005) provide an overview of studies showing the frequencies of occurrence of each learning style. Furthermore, research deals with investigating the reliability and validity of ILS. While some studies conclude that ILS seems to be 
reliable and valid (Felder and Spurlin 2005; Litzinger et al. 2005; Zywno 2003), other studies found limitations in the reliability and validity of the instrument as well as some unexpected dependencies between learning styles (Van Zwanenberg et al. 2000; Viola et al. 2007). Besides these inconsistent results, it is argued that questionnaires have to deal with the problem that the given answers might not correspond to the real behaviour the questions aim to investigate, both intentionally and unintentionally (Draper 1996; Paredes and Rodríguez 2004).

\section{Automatic Learner Modelling Approach}

Instead of asking learners about their preferences, recent research focuses on observing the behaviour of learners during an online course and inferring from this behaviour their learning styles.

Paredes and Rodríguez (2004) introduced a mixed learner modelling approach. In the TANGOW system, learners fill out the ILS questionnaire when they $\log$ in the first time. This information is then used to initialize the learner model. To update and control the information in the learner model, the behaviour of the learners in the system is monitored. If learners behave contrary to the determined learning style preference stored in the learner model, the information in the learner model is revised. TANGOW incorporates only the sensing/intuitive and the sequential/global dimension of FSLSM. Furthermore, only four patterns, each of one learning style preference, are observed for revisions. Since only one corresponding adaptation feature exists per learning style, this approach is suitable for the system to provide appropriate adaptivity. However, it seems to not have enough information to detect completely the learning styles proposed by Felder and Silverman.

Recent research deals with a fully automatic learner modelling approach which considers several patterns per learning style dimension to draw conclusions about the learning style preferences on the respective dimensions. García et al. (in press) proposed an automatic approach for the system SAVER and performed an experiment to show the effectiveness of their approach. The approach considers the active/reflective, sensing/intuitive, and the sequential/global dimensions. The visual/verbal dimension is not incorporated since no relevant learning material is presented in the course. Overall, 11 patterns are included for the three dimensions. Based on the data from these patterns, Bayesian networks (Jensen 1996) are used to calculate the preferences of the learning style dimensions for each learner. The conduced evaluation of the approach shows a high degree of precision when comparing the calculated learning styles of the 10 users with their results from ILS. 
Another approach for automatic learner modelling was investigated by Cha et al. (2006). Again, they observed the behaviour of learners during an online course in an intelligent learning environment based on specific patterns. An experiment with 70 learners was conducted in order to test the effectiveness of Decision Trees (DT) (Dunham 2002) and Hidden Markov Models (HMM) (Rabiner 1989) for detecting learning styles according to FSLSM. For both techniques, several patterns were incorporated for each learning style dimension. However, only data indicating a strong or moderate preference for a specific learning style dimension ( $>3$ according to ILS results) was included in the experiment. While for the visual/verbal dimension, DT achieved the better result, for the sequential/global dimension, the HMM performed better. This can be argued by the fact that HMM are able to consider sequences of learners' actions which might be more relevant for the sequential/global dimension. Results for the sensing/intuitive and the active/reflective dimension were for both techniques the same and the error rates $(22.22 \%$ for sensing/intuitive and $33.33 \%$ for active/reflective) were quite high.

The above approaches are developed for specific systems and therefore used only those patterns for which the system was able to collect data. Graf and Kinshuk (2006a) proposed a tool for detecting learning styles in learning management systems (LMS) in general. The tool extracts relevant data about learners' behaviour from different LMS databases considering that different LMS have different database schemata. The patterns relevant for detecting learning styles were derived from commonly used features in LMS such as forums and exercises. Again, for each learning style dimension several patterns were defined. The calculation of the learning styles is based on the approach used in the ILS questionnaire. Therefore, the gathered data for each pattern indicates either a preference for one or the other style of the dimension, or respectively no preference. As a result, the values of the patterns for each dimension were summed up and scaled. This approach considers that not all LMS might be able to provide data for all patterns. However, the more patterns included in the calculation process, the more stable the result is. For further investigations, the learning management system Moodle (2007) was extended in order to provide all recommended data (Graf and Kinshuk 2006b).

\subsubsection{Relationship between Cognitive Traits and Learning Styles}

So far, cognitive traits and learning styles were discussed separately and information was respectively gathered from the behaviour of learners 
during an online course. As discussed before, the challenge of the automatic learner modelling approach is to identify and collect sufficient information to make reliable and useful inferences. To support the detection process of required information, it is beneficial to find mechanisms that use whatever information about the learner is already available to obtain as much reliable information as possible to build a more robust student model.

In order to get additional information, the relationship between learning styles and cognitive traits was investigated. In web-based educational systems that consider either only learning styles or only cognitive traits, the relationship leads to more information. This additional information can be used to provide better adaptivity, for combined learning styles and cognitive traits instead of only for one of them. In systems that incorporate learning styles as well as cognitive traits, the interaction can be used to improve the detection process of the counterpart. This leads to a more reliable student model. For instance, considering the learner modelling approach for cognitive traits (described in section 5.2.1), the information about learning styles can be treated equally to a Manifestation of Traits. In the neural network, the impact of this new MOT can be calculated and the information can be used for detecting cognitive traits. For learning styles, equally the information about cognitive traits can be seen as a pattern that gives an additional indication for a specific learning style preference.

Graf, Lin, and Kinshuk (in press) investigated the relationship between the Felder-Silverman learning style model and one cognitive trait, namely working memory capacity. They conducted a comprehensive literature review and found several studies indicating a relationship between the four dimensions of FSLSM and working memory capacity. Based on the literature, a relationship between high working memory capacity and a reflective, intuitive, and sequential learning style was identified. In contrast, learners with low working memory capacity tend to prefer an active, sensing, and global learning style. Regarding the visual/verbal dimension, it can be concluded that learners with low working memory capacity tend to prefer a visual learning style but learners with a visual learning style do not necessarily have low working memory capacity.

To verify the proposed relationship, an exploratory study with 39 students was conducted (Graf et al. 2006). The results show that the identified relationship between working memory capacity and two of the four dimensions of the learning style model - the sensing/intuitive and the visual/verbal dimension - was significantly supported. For the two remaining dimensions, only tendencies but no significant correlations were found and therefore, a further study with a larger sample size is planned. 


\subsection{Providing Adaptivity}

In the previous section, we focused on how cognitive traits and learning styles can be detected. In the most cases, the reason for detecting such information and building a learner model is to provide adaptivity. Different possibilities exist for adapting a course based on the information of the learner model. The most often used approach is to match the instructions to the preferences or abilities of the learners and teach according to the learners' strengths. This approach aims at a short-term goal namely to make learning as easy as possible at the time learners are using the system. Looking at long-term goals, Messick (1976) suggested that learners should also train their not-preferred skills and preferences. He argued that when learners acquire more educational experience, they are required to adapt to a variety of instructional methods and styles. The ability to adapt to different instructional styles will prepare them with important life skills. For example, providing verbal learners with only visual forms of instruction forces them to develop and use visual skills.

However, for both approaches the needs of learners have to be identified and then based on this information the respective approach can be chosen. Which approach should be applied might depend on specific conditions such as the current learning goal, the experience of the learner in a particular subject and so on.

In the following two subsections, we discuss how instructions can support specific needs, either learning style preferences or cognitive abilities. Examples are given showing how courses can match (or mismatch) to specific preferences or abilities.

\subsubsection{Adaptivity based on Cognitive Traits}

Humans typically have a number of cognitive abilities. In this subsection, we focus on cognitive abilities which are important for learning. These include working memory capacity, inductive reasoning ability, information processing speed, and associative learning skills. These four cognitive traits are also considered in CTM. For each of these traits Kinshuk and Lin (2003) introduced suggestions on how to support learners with low and high cognitive abilities in adaptive educational systems. These suggestions are based on the Exploration Space Control elements (Kashihara et al. 2000) which are elements that can be changed to create different versions of courses to suit different needs. These elements include the number and relevance of paths, the amount, concreteness and structure of content, as well as the number of information resources. 
Working memory allows us to keep active a limited amount of information (roughly 7+-2 items) for a brief period of time (Miller 1956). Matching courses to the working memory capacity of individual learners aims at considering their abilities and therefore avoiding cognitive overload. For learners with low working memory capacity this can be achieved by decreasing the number and increasing the relevance of paths in a course. Furthermore, less but more concrete content should be presented and the number of available media resources should increase. In contrast, for learners with high working memory capacity, less relevant paths can be presented with the amount of content as well as its abstractness being increased.

Inductive reasoning skills relate to the ability to construct concepts from examples. For learners with low inductive reasoning skills, many opportunities for observation should be provided. Therefore, learning systems can support these learners by providing a high amount of wellstructured and concrete information with many paths. For learners with high inductive reasoning skills, the amount of information and paths should decrease to reduce the complexity of the hyperspace and hence enable the learners to grasp the concepts quicker. Moreover, information can be presented in a more abstract way.

Information processing speed determines how fast the learners acquire the information correctly. For learners with low information processing speed, only the important points should be presented. Therefore, the number of paths and information should decrease and the relevance of paths should increase. Additionally, the structure of the information should increase in order to speed up the learning process. In contrast, for learners with high information processing speed the information space can be enlarged by providing a high amount of information and paths.

The associative learning skills link new knowledge to existing knowledge. In order to assist the association processes during the student's learning, the instruction needs to assist the recall (revisit) of learned information, to clearly show the relationships of concepts (new to existing), and to facilitate new or creative association/insight formation by providing information of the related domain area. High amount of information, different media resources, and many relevant paths help a learner with low associative learning skills to associate one concept to another. Furthermore, well-structured information makes linkage between concepts easier. In contrast, for learners with high associative learning skills less structure of information allows them to navigate more freely and hence enhances the learning speed. Additionally, the relevance of the paths should decrease to enlarge the information space. 


\subsubsection{Adaptivity based on Learning Styles}

Looking at the Felder-Silverman learning style model, some systems exist that provide adaptivity based on dimensions of FSLSM. All these systems aim at providing courses that fit to the learning style preferences of the learners. In the following section, we discuss the possible adaptation features for each dimension of FSLSM.

The sequential/global dimension is often used in adaptive systems. For example, the TANGOW system provides adaptivity by modifying the order of tasks in the course (Paredes and Rodríguez 2004). For a sequential learning style, a more structured path through the learning material is provided whereas global learners are presented with a more open course structure. Bajraktarevic et al. (2003) proposed a system that provides sequential learners with small chunks of text-only information and also hides all links apart from the next and back buttons for navigation in order to provide a more structured path. In contrast, for global learners pages comprised elements such as a table of contents, summary, diagrams, overview of information and so on to give the learner an overall picture. Additionally and in agreement with Paredes and Podríguez, several links within the text were provided in order to provide an open structure. Similarly, Hong and Kinshuk (2004) suggested implementation rules to present the learning material step by step and to constrict links for sequential learners as well as to show global learners the big picture of the course and provide all links for them. The CS383 system (Carver et al. 1999) focused on the order of multimedia objects. For sequential learners, slide shows, hierarchical structured hypertext, and media objects were listed with higher priority, whereas for global learners, lesson objectives, hypertext, a response system, a digital library, and media objects were recommended. Looking at learning management systems, a concept was proposed to present tests and exercises after each learning unit and therefore more frequently for sequential learners, whereas for global learners, these tests and exercises were recommended at the end of the chapter (Graf 2005). Furthermore, it is again suggested to hide links and highlight the back and next buttons for sequential learners and present outlines, show links within the text, and provide additionally a navigation menu for global learners.

Considering the sensing/intuitive dimension, the TANGOW system provides adaptivity by modifying the order of presentation within tasks. For sensing learners, an example is presented first and then the explanation is given, whereas an intuitive learner gets first the explanation and then an example (Paredes and Rodríguez 2004). This feature is also suggested by Hong and Kinshuk (2004). Furthermore, they recommended more hands- 
on activities for sensing learners and more concepts and abstract content for intuitive learners. While the above cited works adapted to the order of examples in the course, Graf (2005) suggested an increase in the number of examples for sensing learners. Furthermore, multimedia objects such as audio objects and interactive animations should be presented to sensing learners. In CS383 (Carver et al. 1999), slide shows, hypertext, a response system, a digital library, and media clips are recommended for sensing learners, whereas for global learners, the learning objectives, slide shows, a response system, and media objects are ranked highly.

Looking at the visual/verbal dimension, most adaptive systems work on the basis of providing visual learners with visual material such as graphics, diagrams, images as well as animations, whereas courses for verbal learners are text-based or include audio objects (Carver et al. 1999; Graf 2005; Hong and Kinshuk 2004). Additionally, communication features are suggested for supporting verbal learners (Graf 2005).

Regarding the active/reflective dimension, Carver et al. (1999) argued that the nature of hypermedia systems inherently supports both active and reflective learning. These systems force students to make choices and look at specific learning material which facilitates active learning. On the other hand, reflective learning is supported since students can reflect and think about the material at any point in their studies. Therefore, no adaptivity regarding the active/reflective dimension is provided in CS383. Graf (2005) suggested providing more multimedia objects such as interactive animations, more exercises as well as communication features for active learners. Hong and Kinshuk (2004) agreed on the communication features and additionally suggested encouraging reflective learners to write summaries about the already learned material.

\subsection{Conclusion}

In this chapter, we provided an overview about learner modelling with focus on cognitive traits and learning styles. We discussed how cognitive traits and learning styles can be identified. Investigations about the relationship between cognitive traits and learning styles were presented, showing how this additional information can be used to improve learner modelling. Since learner modelling enables a system to provide adaptivity, some adaptive features for cognitive traits and learning styles were shown.

More and more recent research is done in modelling and providing adaptivity based on individual characteristics such as cognitive traits and learning styles. However, when having in mind systems that automatically 
detect the individual differences and use this information to provide adaptive instructions concerning short-term and long-term goals of learning, many issues are still open. For instance, further research is necessary on developing a stable approach for automatic learner modelling which is not restricted to a specific system. Another open question concerns the application of different kinds of adaptivity. On the one hand, a short-term goal is to provide students with courses that fit to their needs, makes learning easier for them. On the other hand, long-term goals should be considered by challenging learners with mismatched courses to train their weak abilities and preferences and provide them with important life skills.

\section{References}

Bajraktarevic N, Hall W, Fullick P (2003) Incorporating learning styles in hypermedia environment: Empirical evaluation. In: Proceedings of the Workshop on Adaptive Hypermedia and Adaptive Web-Based Systems, Nottingham, UK, pp 41-52

Brusilovsky P (1994) The construction and application of student models in intelligent tutoring systems. Journal of Computer and Systems Sciences International 32(1):70-89

Brusilovsky P (1996) Methods and techniques of adaptive hypermedia. User Modeling and User-Adapted Interaction 6(2-3):87-129

Carver CA, Howard RA, Lane WD (1999) Addressing different learning styles through course hypermedia. IEEE Transactions on Education 42(1):33-38

Cha HJ, Kim YS, Park SH, Yoon TB, Jung YM, Lee J-H (2006) Learning style diagnosis based on user interface behavior for the customization of learning interfaces in an intelligent tutoring system. In: Ikeda M, Ashley KD, Chan T$\mathrm{W}$ (eds) Proceedings of the 8th International Conference on Intelligent Tutoring Systems, LNCS, vol 4053. Springer-Verlag, pp 513-524

Deary IJ, Whiteman, M.C., Starr, J.M., Whalley, L.J., \& Fox, H.C. (2004) The impact of childhood intelligence on later life: Following up the Scottish mental surveys of 1932 and 1947. Journal of Personality and Social Psychology 86(1):130-147

Draper SW (1996) Observing, measuring, or evaluating courseware: A conceptual introduction. In: Stoner G (ed), Implementating learning technology. Learning Technology Dissemination Initiative, pp 58-65

Dunham MH (2002) Data mining: Introductory and advanced topics. Prentice Hall, Upper Saddle River, NJ, USA

Felder RM, Silverman LK (1988) Learning and teaching styles in engineering education. Engineering Education 78(7):674-681. Preceded by a preface in 2002: http://www.ncsu.edu/felderpublic/Papers/LS-1988.pdf (retrieved July $23,2006)$. 
Felder RM, Soloman BA. (1997). Index of Learning Styles Questionnaire. Retrieved 30 April, 2006, from http://www.engr.ncsu.edu/learningstyles/ ilsweb.html

Felder RM, Spurlin J (2005) Applications, reliability and validity of the Index of Learning Styles. International Journal on Engineering Education 21(1):103112

García P, Amandi A, Schiaffino S, Campo M (in press) Evaluating Bayesian networks' precision for detecting students' learning styles. Computers \& Education

Graf S (2005) Fostering adaptivity in e-learning platforms: A meta-model supporting adaptive courses. In: Proceedings of the IADIS International Conference on Cognition and Exploratory Learning in Digital Age. IADIS Press, pp 440-443

Graf S, Kinshuk (2006a) An approach for detecting learning styles in learning management systems. In: Proceedings of the International Conference on Advanced Learning Technologies. IEEE Computer Science, Alamitos, CA, pp $161-163$

Graf S, Kinshuk (2006b) Considering learning styles in learning management systems: Investigating the behavior of students in an online course. In: Proceedings of the First IEEE International Workshop on Semantic Media Adaptation and Personalization (SMAP 06). IEEE Press, pp 25-30

Graf S, Lin T, Jeffrey L, Kinshuk (2006) An exploratory study of the relationship between learning styles and cognitive traits. In: Proceedings of the European Conference of Technology Enhanced Learning, Lecture Notes in Computer Science 4227. Springer Verlag, Heidelberg, pp 470-475

Graf S, Lin T, Kinshuk (in press) The relationship between learning styles and cognitive traits - getting additional information for improving student modelling. International Journal on Computers in Human Behavior

Honey P, Mumford A (1982) The manual of learning styles. Peter Honey, Maidenhead

Hong H, Kinshuk (2004) Adaptation to student learning styles in web based educational systems. In: Cantoni L, McLoughlin C (eds) Proceedings of World Conference on Educational Multimedia, Hypermedia \& Telecommunications (ED-MEDIA), pp 491-496

Jensen FV (1996) An introduction to Bayesian networks. Springer Verlag, New York

Jonassen DH, Grabowski BL (1993) Handbook of individual differences, learning, and instruction. Lawrence Erlbaum Associates, Hillsdale, New Jersey

Kashihara A, Kinshuk, Oppermann R, Rashev R, Simm H (2000) A cognitive load reduction approach to exploratory learning and its application to an interactive simulation-based learning system. Journal of Educational Multimedia and Hypermedia 9(3):253-276

Kinshuk, Lin T (2003) User exploration based adaptation in adaptive learning systems. International Journal of Information Systems in Education 1(1):2231 
Kinshuk, Lin T (2004) Cognitive profiling towards formal adaptive technologies in web-based learning communities. International Journal of WWW-based Communities 1(1):103-108

Kolb DA (1984) Experiential learning: Experience as the source of learning and development. Prentice-Hall, Englewood Cliffs, New Jersey

Kuljis J, Liu F (2005) A comparison of learning style theories on the suitability for elearning. In: Hamza MH (ed), Proceedings of the IASTED Conference on Web Technologies, Applications, and Services. ACTA Press, pp 191-197

Lin T, Kinshuk (2004) Dichotomic node network and Cognitive Trait Model. In: Proceedings of IEEE International Conference on Advanced Learning Technologies. IEEE Computer Science, Los Alamitos, CA, pp 702-704

Lin T, Kinshuk (2005) Cognitive profiling in life-long learning. In: Howard C, Boettcher JV, Justice L, Schenk K, Rogers PL, Berg GA (eds) Encyclopedia of International Computer-Based Learning. Idea Group Inc., Hershey, PA, USA, pp 245-255

Litzinger TA, Lee SH, Wise JC, Felder RM (2005) A study of the reliability and validity of the Felder-Soloman Index of Learning Styles. In: Proceedings of the ASEE Annual Conference. American Society for Engineering Education

Messick S (1976) Personal styles and educational options. In: Messick S (ed), Individuality in learning. Jossey Bass, San Francisco, pp 327-368

Miller GA (1956) The magic number seven, plus or minus two: Some limit of our capacity for processing information. Psychology Review 63(2):81-96

Moodle. (2007). Retrieved 6 January, 2007, from http://www.moodle.org

Paas F, Renkl A, Sweller J (2003) Cognitive load theory and instructional design: Recent developments. Educational Psychologist 38:1-4

Paredes P, Rodríguez P (2004) A mixed approach to modelling learning styles in adaptive educational hypermedia. Advanced Technology for Learning 1(4):210-215

Rabiner LR (1989) A tutorial on hidden Markov models and selected applications inspeech recognition. Proceedings of the IEEE 77(2):257-286

Self J (1994) Formal approaches to student modelling. In: McCalla GI, Greer J (eds) Student modelling: The key to individualized knowledge-based instruction. Springer, Berlin, pp 295-352

Sweller J (1988) Cognitive load during problem solving. Cognitive Science 12:257-285

Van Zwanenberg N, Wilkinson LJ, Anderson A (2000) Felder and Silverman's Index of Learning Styles and Honey and Mumford's Learning Styles Questionnaire: How do they compare and do they predict academic performance? Educational Psychology 20(3):365 - 380

Viola SR, Graf S, Kinshuk, Leo T (2007) Investigating relationships within the Index of Learning Styles: A data-driven approach. International Journal of Interactive Technology and Smart Education 4(1):7-18

Zywno MS (2003) A contribution to validation of score meaning for FelderSoloman's Index of Learning Styles. In: Proceedings of the ASEE Annual Conference. American Society for Engineering Education 